\title{
G Research Square $=1.5$ \\ Intermediate-dose cytarabine is an effective therapy for adults with non-Langerhans cell histiocytosis
}

\section{Ting Liu}

PUMCH: Peking Union Medical College Hospital

\section{Hua-cong Cai}

PUMCH: Peking Union Medical College Hospital

Hao Cai

PUMCH: Peking Union Medical College Hospital

\section{Miao Chen}

PUMCH: Peking Union Medical College Hospital

Wei Zhang

PUMCH: Peking Union Medical College Hospital

Jian Li

PUMCH: Peking Union Medical College Hospital

Dao-bin Zhou

PUMCH: Peking Union Medical College Hospital

Xin-xin Cao ( $\sim$ caoxinxin@pumch.cn )

Peking Union Medical College Hospital https://orcid.org/0000-0001-7884-3073

\section{Research}

Keywords: Non-Langerhans Cell Histiocytosis, Cytarabine, Efficacy

Posted Date: November 19th, 2021

DOI: https://doi.org/10.21203/rs.3.rs-1064331/v1

License: (c) (1) This work is licensed under a Creative Commons Attribution 4.0 International License. Read Full License

Version of Record: A version of this preprint was published at Orphanet Journal of Rare Diseases on February 7th, 2022. See the published version at https://doi.org/10.1186/s13023-022-02193-0. 


\section{Abstract \\ Background}

Non-Langerhans cell histiocytosis, including Erdheim-Chester disease (ECD), Rosai-Dorfman disease (RDD), indeterminate cell histiocytosis (ICH), and unclassified histiocytosis, is a rare disorder lacking a standard treatment strategy. We report our experience using intermediate-dose cytarabine as the first or subsequent therapy in non-Langerhans cell histiocytosis.

\section{Results}

Eight ECD patients, 5 RDD patients, $1 \mathrm{ICH}$ patient and 1 unclassified histiocytosis patient were enrolled. Intermediate-dose cytarabine therapy was administered as $0.5-1.0 \mathrm{mg} / \mathrm{m}^{2}$ of intravenous cytarabine every 12 hours for 3 days every 5 weeks. The median age at cytarabine initiation was 45 years (range, 18-70 years). The median number of cycles of cytarabine administered was 6 (range, 2-6). The overall response rate (ORR) was $86.7 \%$ in the overall cohort, including $6.7 \%$ with complete response and $80.0 \%$ with partial response. All patients $(n=10)$ with CNS involvement achieved disease improvements. One patient experienced disease recurrence 19 months after cytarabine therapy. The median follow-up duration for the entire cohort was 12 months (range, 4-61 months). The 1-year progression-free survival (PFS) and overall survival (OS) rates were $85.6 \%$ and $92.3 \%$, respectively. The most common toxicity was haematological adverse events, including grade 4 neutropenia and grade $3-4$ thrombocytopenia. No treatment-related deaths occurred.

\section{Conclusions}

Intermediate-dose cytarabine is a cost-effective treatment option for non-Langerhans cell histiocytosis patients, especially for those with CNS involvement.

\section{Introduction}

Histiocytosis is a rare disorder characterized by the accumulation of macrophages, dendritic cells, or monocyte-derived cells in various tissues and organs[1]. Historically, histiocytic neoplasms have been classified into Langerhans cell histiocytosis ( $(\mathrm{CH})$ and non-Langerhans cell histiocytosis, including Erdheim-Chester disease (ECD), juvenile xanthogranuloma (JXG), Rosai-Dorfman disease (RDD), and indeterminate cell histiocytosis (ICH) [2]. The most common types of non-Langerhans cell histiocytosis are ECD and RDD. Their heterogeneity and rarity pose great challenges to the establishment of standard treatment strategies.

The discovery of the BRAF ${ }^{\mathrm{V} 600 \mathrm{E}}$ mutation in approximately $50 \%$ of patients with $\mathrm{LCH}[3]$ and $\mathrm{ECD}[4]$ provided the first molecular therapeutic target in histiocytosis. BRAF inhibition is highly efficacious and 
has markedly altered the natural history of these disorders[5]. For patients who lack BRAF ${ }^{V 600}$ mutations but carry other MAPK-ERK pathway mutations, treatment with MEK inhibitors has shown clinical efficacy[6]. However, recurrent driving mutations of the MAPK/ERK pathway are not universal in nonLangerhans cell histiocytosis, and recent biological and molecular advances in ECD have not been matched in other non-Langerhans disorders. For non-Langerhans cell histiocytosis types excluding ECD, the efficacy of targeted therapy has only been reported in isolated case reports.

Cytarabine is a cost-effective cytotoxic drug that plays an important role in the treatment of haematological neoplasms, including histiocytic neoplasms. We previously reported remarkable responses to intermediate-dose cytarabine in 3 patients with $\operatorname{ECD}[7,8]$ and 2 patients with $\operatorname{RDD}[9]$ with central nervous system (CNS) involvement.

Here, we conduct a retrospective review of the use of intermediate-dose cytarabine in adults with nonLangerhans cell histiocytosis, including ECD, RDD, ICH and unclassified histiocytosis, to analyse the efficacy and safety of cytarabine in these patients.

\section{Methods}

\section{Patients}

A retrospective review was conducted among patients who were diagnosed with non-Langerhans cell histiocytosis (ECD, RDD, ICH and unclassified histiocytosis) and had received intermediate-dose cytarabine for at least 2 cycles at Peking Union Medical College Hospital between October 2013 and August 2021. The diagnosis of non-Langerhans cell histiocytosis was based on typical clinical presentation, radiologic presentation, and histologic findings that were reviewed independently by two pathologists. Informed consent was obtained from all patients, and the protocol was approved by the Peking Union Medical College Hospital Ethics Committee. The present study was performed in accordance with the ethical standards of the 1964 Declaration of Helsinki and its later amendments.

\section{Clinical, imaging, and genetic data}

Clinical data were collected regarding age, sex, lesion location, physical examination, routine biologic analysis, treatment, and survival. Imaging data were collected from FDG-PET; computed tomography (CT) of the entire aorta, chest, abdomen and pelvis; and magnetic resonance imaging (MRI) of the brain and heart. DNA extracted from formalin-fixed and paraffin-embedded preserved lesion biopsy samples of the enrolled patients was obtained and subjected to next-generation sequencing of 183 genes as previously described[10]. The presence of the BRAF ${ }^{\mathrm{V} 600 \mathrm{E}}$ mutation was detected by polymerase chain reaction (PCR) in some cases as previously described[11].

\section{Treatment, response and toxicity criteria}

Intermediate-dose cytarabine therapy was defined as the administration of $0.5-1.0 \mathrm{mg} / \mathrm{m}^{2}$ of intravenous cytarabine every 12 hours for 3 days every 5 weeks for $4-6$ cycles in total. All patients were followed up 
every 3-6 months. Response assessment was primarily performed using the PET Response Criteria in Solid Tumours (PERCIST)[12], and the patients were then classified as having complete metabolic response (CMR, complete resolution of pathologic FDG uptake), partial metabolic response (PMR, reduction of a minimum of $30 \%$ in activity of the target lesions), stable metabolic disease (SMD, not complete or partial metabolic response), or progressive metabolic disease (PMD, increase of a minimum of $30 \%$ in the activity of the target lesions or the presentation of a new lesion). Patients unable to undergo FDG-PET underwent response assessment using CT or MRI according to the Response Evaluation Criteria in Solid Tumors (RECIST; version 1.1) [13]. Responses were categorized as follows: complete response (CR): disappearance of all target lesions; partial response (PR): at least a $30 \%$ decrease in the sum of the diameters of the target lesions; progressive disease (PD): at least a $20 \%$ increase in the sum of the diameters of the target lesions; and stable disease (SD): neither sufficient shrinkage to qualify for PR nor sufficient increase to qualify for PD. Chemotherapy-related toxicities were assessed using the Common Terminology Criteria for Adverse Events (CTCAE) version 4.03 (National Cancer Institute, Bethesda, MD).

\section{Statistical analysis}

The follow-up was conducted up to October 31, 2021. Overall survival (OS) was defined as the time from the date of cytarabine treatment to the date of death or the last follow-up. Progression-free survival (PFS) was calculated from the date of cytarabine treatment until the date of disease progression, relapse, or death from any cause. We performed all statistical analyses using SPSS version 21 (IBM Corp., Armonk, NY, USA). Kaplan-Meier analysis was used for survival analysis, with the survival curves compared using the log-rank test.

\section{Results}

\section{Patients}

A total of 15 patients ( 9 males and 6 females) met the inclusion criteria. The patients were diagnosed with $\operatorname{ECD}(n=8), \operatorname{RDD}(n=5), I C H(n=1)$ and unclassified histiocytosis $(n=1$, Table 1$)$. The median age at cytarabine initiation was 45 years (range, $18-70$ years). Twelve $(75.0 \%)$ patients had multisystem disease, and the most commonly involved organs were the bones (73.3\%), CNS (66.7\%), orbit $(26.7 \%)$ and retroperitoneum (including the kidneys, $26.7 \%)$, thyroid (20.0\%), and skin (13.3\%).

We performed next-generation sequencing on 6 patients with ECD and 2 patients with RDD. No pathogenic mutations were detected in 3 patients with ECD, and the remaining 3 patients had the $\mathrm{BRAF}^{\mathrm{V} 600 \mathrm{E}}$ mutation. Mutations of $\mathrm{BRAF}^{\mathrm{R} 188 \mathrm{G}}$ at a variant-allele frequency of $4.3 \%$ and MAP2K1 ${ }^{\mathrm{D} 147 \mathrm{G} 1}$ at a variant-allele frequency of $5.4 \%$ were detected in one RDD patient, while no pathogenic mutations were detected in the other RDD patient. The BRAF ${ }^{\mathrm{V} 600 \mathrm{E}}$ status was detected by PCR in 2 patients (1 ECD and 1 $\mathrm{RDD}$ ), and a patient with $\mathrm{ECD}$ had the $\mathrm{BRAF}^{\mathrm{V} 600 \mathrm{E}}$ mutation, while another patient was $\mathrm{BRAF}^{\mathrm{V} 600 \mathrm{E}}$ wild type.

\section{Treatment and response}


Cytarabine was administered as frontline systemic therapy in 8 (53.3\%) patients and as subsequent-line treatment in $7(46.7 \%)$ patients. Of the 7 patients who received cytarabine in the subsequent line, prior therapies included interferon- $a(n=3)$, polychemotherapy $(n=2)$, corticosteroids $(n=1)$, and surgery $(n=1$, Table 1). The median number of cycles of cytarabine administered was 6 (range, 2-6). Response assessment was conducted using FDG-PET in 9 (60.0\%) patients, and the response rates were as follows: CMR, 11.1\% $(n=1)$; PMR, 77.8\% $(n=7)$; and PMD, 11.1\% $(n=1)$. The remaining 6 patients were assessed by CT or MRI; 5 patients achieved PR (83.3\%), and 1 patient had PD and died. Therefore, the overall response rate (ORR) was $86.7 \%(n=13)$ in the overall cohort. All patients with CNS involvement $(n=10)$ achieved disease improvements.

\section{Survival and toxicity}

The median follow-up duration for the entire cohort was 12 months (range, 4-61 months). After cytarabine therapy, 8 patients received interferon-a (IFN- $a$ ), and 2 patients received lenalidomide plus dexamethasone for maintenance. One patient with ECD experienced recurrence during the maintenance treatment of IFN-a (19 months after cytarabine therapy) and was switched to vemurafenib therapy. The patient who did not respond to cytarabine therapy was then treated with sirolimus and prednisone, and the patient's condition was stable up to the last follow-up. The 1-year PFS and OS rates were $85.6 \%$ and 92.3\%, respectively (Figure 1).

The most common toxicity was haematological adverse events. All patients experienced grade 4 neutropenia, and 9 patients experienced neutropenic fever. Eight patients developed grade 3-4 thrombocytopenia, but no severe bleeding events occurred. No treatment-related deaths occurred.

\section{Discussion}

Non-Langerhans cell histiocytosis is a rare disorder lacking a standard treatment strategy. Due to the discovery of activating and targetable MAPK-ERK pathway mutations in the vast majority of patients with ECD, the therapeutic landscape of ECD has changed drastically over the last decade[4,5]. BRAF inhibitors, such as vemurafenib or dabrafenib, are recommended as first-line therapy for patients with multisystem BRAF-V600-mutant ECD who have life-threatening cardiac or neurologic involvement; for patients without BRAF-V600 mutation, NGS is suggested to evaluate other MAPK-ERK pathway alterations that can be treated with a MEK inhibitor[14]. However, BRAF and MEK inhibitors are costly (far beyond what most patients in China can afford) and are not covered by health insurance in China.

Treatment is reserved for symptomatic disease or multisystemic involvement for patients with RDD. The conventional systemic therapies for RDD include steroids, chemotherapy, sirolimus, and immunomodulatory therapy, such as thalidomide and lenalidomide[15]; however, the efficacy of steroids or other systemic therapies for RDD is variable. MEK inhibitors such as cobimetinib used in patients with MAPK-ERK pathway alterations seem promising[6], but have limited experience. Data regarding the efficacy and safety of systemic treatments for other non-Langerhans cell histiocytic disorders, such as ICH and JXG, are especially lacking. Overall, the management of patients with non-Langerhans cell 
histiocytosis is challenging. Systemic nontargeted therapy for patients with non-Langerhans cell histiocytosis deserves exploration.

Our previous data suggested that CNS involvement was a poor prognostic factor for ECD patients with interferon-a-2a (IFN-a)[16]. Therefore, we attempted to explore treatments other than IFN-a for nonLangerhans cell histiocytosis, especially drugs that can penetrate the blood-brain barrier. Cytarabine easily penetrates the blood-brain barrier and has shown promising therapeutic prospects in nonLangerhans cell histiocytic disorders[8, 9].

The patients enrolled in the present study had multisystem involvement with or without previous therapy. Most relapsed patients in this study accepted standard first-line treatments, as reported in the literature $[14,15]$. Although some patients in our study carried the BRAF ${ }^{\mathrm{V} 600 \mathrm{E}}$ mutation, they could not afford BRAF inhibitors as first-line treatment. We found that cytarabine has favourable clinical efficacy in non-Langerhans cell histiocytosis patients with multisystem involvement regardless of its use as frontline therapy or subsequent-line treatment. The overall clinical response rate was $86.7 \%$, with 1 -year PFS and OS rates of $85.6 \%$ and $92.3 \%$, respectively. Impressive responses were seen in patients with central nervous system involvement.

In terms of regimen toxicity, grade 4 neutropenia was noted in all patients, and grade 3-4 thrombocytopenia occurred in $53.3 \%$ of patients during treatment. Nevertheless, no treatment-related deaths occurred. It is critical to monitor routine blood tests regularly and use granulocyte colonystimulating factor, granulocyte stimulating factor or transfuse platelets according to routine blood results.

This study's limitations include the small number of patients, the retrospective nature of the analysis, and the lack of long-term follow-up for outcomes and toxicity. Nevertheless, large-scale cohort studies or prospective clinical trials are of great difficulty due to the rarity of the disease. We will extend the followup duration to monitor the long-term outcomes and toxicity.

\section{Conclusion}

In conclusion, intermediate-dose cytarabine is an efficient and safe treatment option for non-Langerhans cell histiocytosis, especially for patients with CNS involvement.

\section{Abbreviations}

CNS: Central nervous system; CMR: complete metabolic response; CR: Complete response; CT: Computed tomography; CTCAE: Common Terminology Criteria for Adverse Events; ECD: Erdheim-Chester disease; FDG-PET:18Ffluorodeoxyglucose positron emission tomography; ICH: indeterminate cell histiocytosis; IFN-a: Interferon-a; LCH: Langerhan s cell histiocytosis; JXG: juvenile xanthogranuloma; MRI: Magnetic resonance imaging; PCR: Polymerase chain reaction; PERCIST: PET Response Criteria in Solid Tumors; PD: progressive disease; PFS: Progression-free survival; PR: Partial response; PMR: partial metabolic response; PMD: progressive metabolic disease; RECIST: Response Evaluation Criteria in Solid Tumors; 
RDD: Rosai-Dorfman disease; ORR: Overall response rate; OS: Overall survival; SD: Stable disease; SMD: stable metabolic disease

\section{Declarations}

\section{Acknowledgments}

The authors thank the patients and their families.

Funding: This work was supported by institutional research funding provided by the Beijing Natural Science Foundation (Grant No. 7202160 to Cao XX), the Non-profit Central Research InstituteFund of the Chinese Academy of Medical Sciences (Grant No. 2019-RC-HL-001 to Cao XX).

\section{Availability of data and materials}

The datasets used and/or analysed during the current study are available from the corresponding author on reasonable request.

\section{Authors' contributions}

Contribution: XC, DZ and JL contributed to the conception and design of the study; $\mathrm{HC}$ contributed to the gene mutation examination; $\mathrm{XC}, \mathrm{HC}, \mathrm{WZ}$ and $\mathrm{MC}$ contributed to the treatment and assessment of the patients; TL contributed to the data analysis and wrote the paper; all authors revised the paper and approved the submitted version.

\section{Ethics approval and consent to participate}

The study protocol was approved by Peking Union Medical College Hospital Ethics Committee. Informed consent was obtained from the participants in accordance with the Declaration of Helsinki.

\section{Consent for publication}

Not applicable.

\section{Competing interests}

The authors declare that they have no competing interests.

\section{References}

1. Emile J-F, Abla O, Fraitag S, et al: Revised classification of histiocytoses and neoplasms of the macrophage-dendritic cell lineages. Blood.2016;127:2672-81.

2. Histiocytosis syndromes in children. Writing Group of the Histiocyte Society. Lancet (London, England).1987;1:208-9. 
3. Badalian-Very G, Vergilio J-A, Degar BA, et al: Recurrent BRAF mutations in Langerhans cell histiocytosis. Blood.2010;116:1919-23.

4. Julien H: High prevalence of BRAF V600E mutations in Erdheim-Chester disease but not in other nonLangerhans cell histiocytoses. Blood. 2012; 120:2700-3.

5. Diamond EL, Subbiah V, Lockhart AC, et al: Vemurafenib for BRAF V600-Mutant Erdheim-Chester Disease and Langerhans Cell Histiocytosis: Analysis of Data From the Histology-Independent, Phase 2, Open-label VE-BASKET Study. JAMA Oncol.2018;4:384-8.

6. Diamond EL, Durham BH, Ulaner GA, et al: Efficacy of MEK inhibition in patients with histiocytic neoplasms. Nature.2019; 567:521-4.

7. Cao X-X, Niu N, Sun J, et al: Efficacy of intermediate-dose cytarabine in central nervous systemrelapsed wild-type BRAF Erdheim-Chester disease. Annals of hematology.2018;97:185-7.

8. Wang J-N, Qiu Y, Niu N, et al: Successful treatment of central nervous system involved ErdheimChester disease by intermediate-dose cytarabine as first-line therapy. Acta oncologica (Stockholm, Sweden). 2020; 59:302-5.

9. Wang W, Sun J, Zhang W, et al: Successful treatment of intracranial Rosai-Dorfman disease with cytarabine and dexamethasone: case report and review of literature. Annals of hematology.2020; 99:1157-9.

10. Chen J, Zhao A-L, Duan M-H, et al: Diverse kinase alterations and myeloid-associated mutations in adult histiocytosis. Leukemia.2021; doi: 10.1038/s41375-021-01439-3

11. Cao X-X, Sun J, Li J, et al: Evaluation of clinicopathologic characteristics and the BRAF V600E mutation in Erdheim-Chester disease among Chinese adults. Annals of hematology.2016;95:745-50.

12. Wahl RL, Jacene H, Kasamon Y, et al: From RECIST to PERCIST: Evolving Considerations for PET response criteria in solid tumors. J Nucl Med 2009;50 Suppl 1:122S-150S.

13. Eisenhauer EA, Therasse $P$, Bogaerts $J$, et al: New response evaluation criteria in solid tumours: revised RECIST guideline (version 1.1). Eur J Cancer.2009;45:228-47.

14. Goyal G, Heaney ML, Collin M, et al: Erdheim-Chester disease: consensus recommendations for evaluation, diagnosis, and treatment in the molecular era. Blood.2020; 135:1929-45.

15. Abla O, Jacobsen E, Picarsic J, et al: Consensus recommendations for the diagnosis and clinical management of Rosai-Dorfman-Destombes disease. Blood.2018;131:2877-90.

16. Cao X-X, Niu N, Sun J, et al: Clinical and positron emission tomography responses to long-term highdose interferon-a treatment among patients with Erdheim-Chester disease. Orphanet journal of rare diseases.2019;14:11.

\section{Tables}

Due to technical limitations, Table 1 is only available as a download in the Supplemental Files section.

\section{Figures}




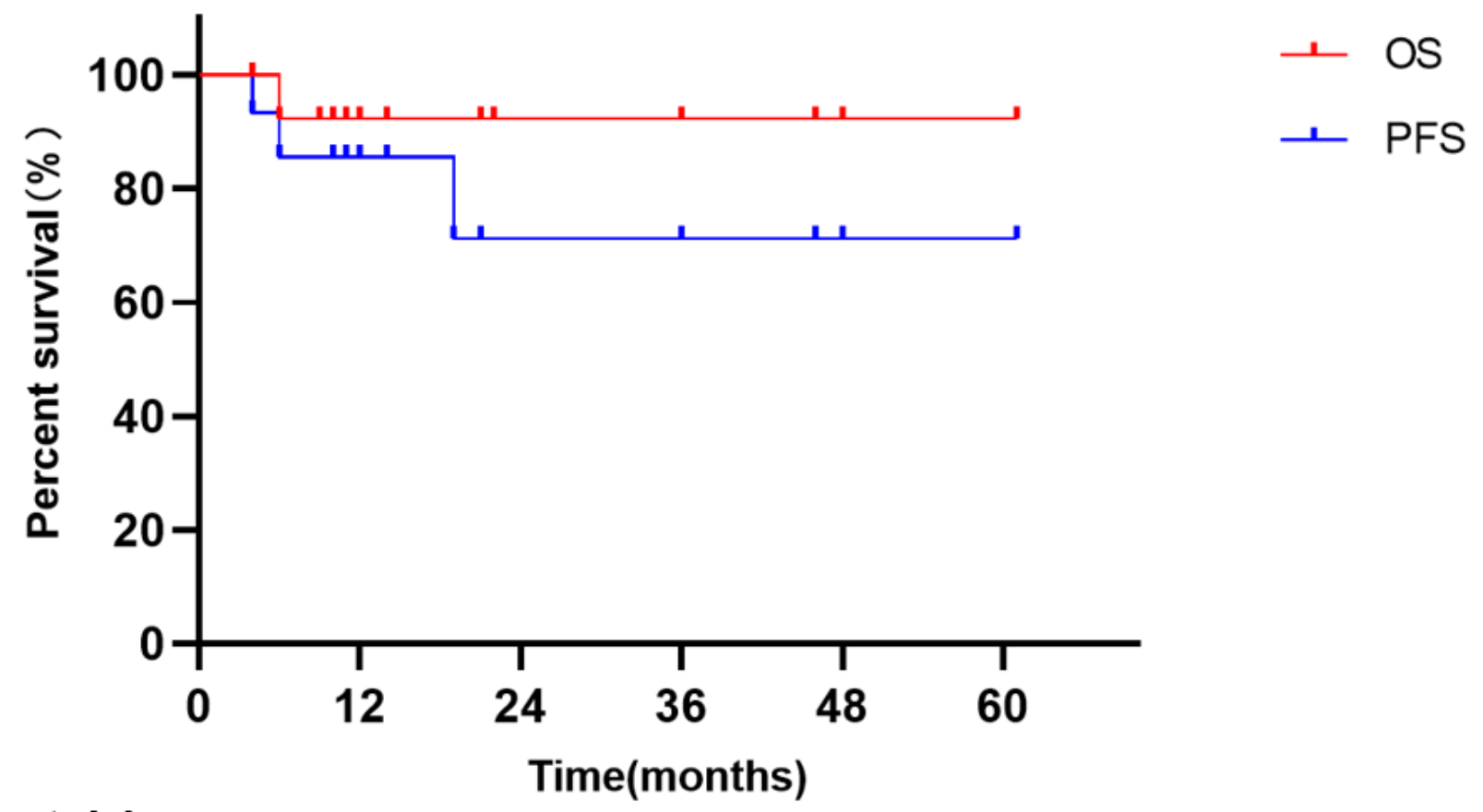

No.at risk

$\begin{array}{lllllll}\text { OS,n } & 15 & 8 & 5 & 4 & 2 & 1 \\ \text { PFS,n } & 15 & 8 & 5 & 4 & 2 & 1\end{array}$

Figure 1

Progression-free survival (PFS) and overall survival (OS) for the whole cohort ( $n=15)$

\section{Supplementary Files}

This is a list of supplementary files associated with this preprint. Click to download.

- Table1.docx 